\title{
Instrumental characterization of the smectite clay-gentamicin hybrids
}

\author{
ALICJA RAPACZ-KMITA ${ }^{1, *}$, EWA STODOLAK-ZYCH ${ }^{1}$, MAGDALENA ZIABKA ${ }^{1}$, \\ AGNIESZKA ROZYCKA ${ }^{1}$ and MAGDALENA DUDEK ${ }^{2}$ \\ ${ }^{1}$ AGH University of Science and Technology, Faculty of Materials Science and Ceramics, Cracow 30-059, Poland \\ ${ }^{2}$ AGH University of Science and Technology, Faculty of Energy and Fuels, Cracow 30-059, Poland
}

MS received 23 January 2015; accepted 4 March 2015

\begin{abstract}
This paper focusses on the intercalation of clay mineral with gentamicin (an aminoglycoside antibiotic). The smectite clay-gentamicin hybrids were prepared by a solution intercalation at $60^{\circ} \mathrm{C}$ and the process was carried out on unmodified smectite clay and on smectite after $\mathrm{Na}^{+}$ionic activation. The resulting structural/microstructural properties and the potential for introducing gentamicin between smectite clay layers were investigated by means of X-ray diffraction, Fourier transform infrared spectroscopic techniques and transmission electron microscopy and scanning electron microscopy with energy-dispersive spectroscopy X-ray analysis. The results confirm the successful intercalation of gentamicin into the interlayer space of smectite clay, demonstrating that the material thus obtained could potentially be used as a drug carrier.
\end{abstract}

Keywords. Clay mineral; drug carrier; intercalation; XRD; FTIR.

\section{Introduction}

Clay and clay minerals are commonly used in the pharmaceutical industry today as both excipients and active substances, and play an important role, not only in pharmaceuticals, but also in other health products. However, the potential benefits of drug therapy were generally not known until the late 1500s, when the Swiss physician Paracelsus pioneered the initial use of minerals as drug therapies. ${ }^{1}$

Nowadays, clays can be considered as raw pharmaceutical materials that may, once evaluated and/or modified to fulfil regulatory pharmacopoeial requirements, achieve the status of pharmaceutical substances suitable for use in the manufacture of medical products. ${ }^{2}$ The high capacity for cation exchange of clays, along with their non-toxicity, high level of biocompatibility and ability to control drug release, ${ }^{3,4}$ makes them attractive candidates for drug carriers. The lastmentioned function is particularly interesting, given that the use of conventional drugs may lead to fluctuations whereby drug concentrations in the organism may reach levels lower than the minimum effective (MEC) or higher than the minimum toxic (MTC) concentrations. This can result in undesirable side effects or failure to deliver therapeutic benefits intended for the patient. In such cases, the use of materials that modify the release of drugs helps to reduce undesirable levels of these fluctuations, decreasing side effects and/or enhancing the drug's therapeutic effect, improving the patient's compliance with the treatment and adding commercial value to medicines sold through extension of patent protection. ${ }^{5,6}$

\footnotetext{
*Author for correspondence (kmita@agh.edu.pl)
}

Many studies have been reported on montmorillonite-drug hybrids for modified drug-delivery systems (MDDS), e.g., ibuprofen, vitamin B1, nicotine, sertraline (an antidepressive drug) and others. ${ }^{7-11}$ The most recent include ibuprofen release control through interaction with montmorillonite ${ }^{7}$ and intercalation of donepezil, a well-known drug for Alzheimer's disease, in montmorillonite, saponite or laponite. ${ }^{12}$ These studies characterized the products of clay mineral-drug interaction and then examined the kinetics of drug release, which, in all cases, was dependent on the method of preparation of the interaction products and was affected by changes in the $\mathrm{pH}$ of the dissolution medium. In the case of donezepil, biphasic release patterns were found, consisting of an initial burst effect followed by slow release of the drug.

Among others, magnesium aluminium silicate (MAS), a mixture of natural smectites, specifically montmorillonite and a low percentage of saponite, has been also studied as a drug carrier of nicotine ${ }^{9}$ and timolol. ${ }^{8}$ The use of MAS as a drug adsorbent to improve physical characteristics and modulate drug release behaviour is motivated by its high surface area, good affinity with a positively charged drug, non-toxicity and non-irritation at the levels employed in pharmaceutical use. ${ }^{13,14}$ Nicotine, a volatile liquid widely used in smoking cessation therapy to relieve addiction symptoms, has been carried by magnesium aluminium silicate to reduce drug evaporation and modulate drug release behaviour. The amount of the drug retained and the release patterns were determined by $\mathrm{pH}$; cation exchange seemed to be the principal interaction mechanism. Timolol has been loaded on montmorillonite, but release of the drug failed to reach $100 \%$. Magnesium aluminium silicate has been also 
used to retain propranolol (a $\beta$-blocking agent) and release patterns have been correlated to particle size, drug loading and release medium. ${ }^{15}$

This paper describes an attempt to intercalate magnesium aluminium silicate smectite clay with gentamicin, which is one of the few thermally stable antibiotics, remaining active even after the autoclaving process. The intercalation process was monitored by X-ray diffraction (XRD) and Fourier transform infrared (FTIR) analysis. The potential for using scanning electron microscopy (SEM) and transmission electron microscopy (TEM) (with energy-dispersive spectroscopy (EDS) X-ray analysis) to assess the degree of intercalation was also taken under consideration. The study aimed at observation of the behaviour of gentamicin in the presence of smectite, as well as determination of the capacity of gentamicin to fill the gaps present in the interlayer spaces of smectite.

\section{Experimental}

\subsection{Materials}

Smectite clay, specifically magnesium aluminium silicate Veegum ${ }^{\circledR} F(V F)$, a white, biologically pure smectite used in the pharmaceutical and cosmetic industries manufactured by R.T. Vanderbilt Company, Inc. (data sheet Vanderbilt Co.), was used in the study as a drug carrier. Gentamicin $(\mathrm{G})$, in the form of gentamicin sulphate $\left(\mathrm{C}_{21} \mathrm{H}_{43} \mathrm{~N}_{5} \mathrm{O}_{7} \cdot \mathrm{H}_{2} \mathrm{SO}_{4}\right)$, was purchased from Sigma-Aldrich USA. Gentamicin belongs to the aminoglycoside family of antibiotics used in medical practice as sulphate in the form of eye ointments or drops and intramuscular injections, and is in fact one of the few thermally stable antibiotics, remaining active even after the autoclaving process.

The intercalation process using gentamicin was done on both unmodified smectite VF (neat product as received from the manufacturer) and VF smectite preliminarily modified/ activated with $\mathrm{Na}^{+}$ions using $\mathrm{NaCl}$ solution. The intercalation of gentamicin into structure of the clay materials was done according to the procedure described in the following section.

The following abbreviations for the materials are used in the study: G-gentamicin, VF-Veegum ${ }^{\circledR}$ F, VFGVeegum ${ }^{\circledR} F$ hybrid intercalated with gentamicin, VFAVeegum ${ }^{\circledR}$ F after ion activation and VFAG - Veegum ${ }^{\circledR} F$ after ion activation hybrid intercalated with gentamicin.

\subsection{Preparation of the smectite-gentamicin hybrids}

The smectite powder was weighed in the amount of $2 \mathrm{~g}$, then mixed with $50 \mathrm{ml}$ of distilled water using a magnetic stirrer while being heated at $60^{\circ} \mathrm{C}$ for $24 \mathrm{~h}$. To the prepared suspensions of the powder, $0.5 \mathrm{~g}$ of gentamicin sulphate was then added to obtain a $4: 1$ smectite/G ratio. After the addition of gentamicin, the solution was mixed again with a magnetic stirrer at $60^{\circ} \mathrm{C}$ for 24 more hours. The thoroughly mixed solution was placed in sterile containers, then quenched with liquid nitrogen and lyophilized in a freeze dryer (FreeZone 6 litre, Labconco) at $-51^{\circ} \mathrm{C}$. The process of freeze-drying was carried out at a pressure of $40 \mathrm{~Pa}$ for $48 \mathrm{~h}$. After intercalation with gentamicin, the powders were washed with distilled water to remove the quantity of the drug that could not be incorporated between the layers. The VF starting powder (unmodified) was also lyophilized after being mixed with water and separated by ultrasonic stirring.

The above procedure was applied to both the nonactivated (VF) and activated (VFA) smectites; however, the latter material had undergone prior modification with $\mathrm{Na}^{+}$ions, using the following process. The VF powder was disperzed in $2 \mathrm{M} \mathrm{NaCl}$ solution, stirred for $24 \mathrm{~h}$ at $50^{\circ} \mathrm{C}$, centrifuged, and rinsed with deionized water up to the extinction of the chlorine ion reaction $\left(\mathrm{AgNO}_{3}\right)$. The obtained powder was then lyophilized. This preliminary modification/activation was expected to provide an answer to the question of whether, given the same amount of the drug to be fed, modified smectite enables the introduction of greater amounts of the drug between the layers.

\subsection{Methods}

The lyophilized powders of smectite clay intercalated with gentamicin (VFG and VFAG), the starting smectite powder (VF and VFA), and the neat gentamycin (G) were examined by XRD, FTIR, TEM and SEM (+ EDS X-ray analysis). The study aimed at observing the behaviour of gentamicin in the presence of smectite and identifying possible ways for gentamicin to be incorporated into the interlayer spaces of smectite (to assess the effectiveness of the intercalation process).

The XRD analysis was performed in the range of $3<2 \theta<70$ with $\mathrm{Cu} \mathrm{K} \alpha$ radiation using a PANalytical Empyrian diffractometer with a step of $0.008^{\circ}$ (total time about $4 \mathrm{~h}$ ). The analysis was focused on the $5<2 \theta<24$ range, where the most characteristic diffraction peaks were expected. The FTIR spectra were recorded by a FTS-60V Bio-Rad spectrometer in the range of $400-4000 \mathrm{~cm}^{-1}$ using $\mathrm{KBr}$ pellets. Microstructural observations of the studied smectite and gentamicin powders were made with SEM (Nova Nano SEM 200, FEI Company and JEOL JSM 5400) using secondary electron image and EDS X-ray analysis (EDAX detector). All types of powders were covered with a carbon layer prior to observation. SEM elemental mapping was carried out using samples in the form of pellets made of all types of powders, pressed under a pressure of $50 \mathrm{MPa}$. Measurements were done under high vacuum conditions using an acceleration voltage of $10 \mathrm{kV}$. Each sample was mapped for the same amount of time with the same amount of counts (100 scans).

Microstructural observations were made with a transmission electron microscope (TEM) (JEOL JEM-1011). EDS analysis was used to investigate the effectiveness of the intercalation process in microregions. All types of powders were dispersed in isopropanol and covered with a carbon layer prior to observation. 


\section{Results and discussion}

\subsection{XRD analysis}

Figure 1 shows the XRD patterns of the pristine montmorillonite and montmorillonite activated with $\mathrm{Na}^{+}$cations (VF and VFA) and compares it with that of montmorillonites that were previously dispersed in a gentamicin sulphate solution (VFG and VFAG). The changes in basal distance of the powders are summarized in table 1 . The diffraction pattern of pure gentamicin which is highly amorphous is also shown for information. The diffractogram of pristine MAS (VF) shows the primary reflection at $7.22^{\circ} 2 \theta$, which corresponds to a basal spacing $d_{001}$ of $12.3 \AA$. The obtained X-ray pattern is in agreement with other reports on magnesium aluminium silicate powders having a water monolayer in the interlayer space. ${ }^{16,17}$ After activation of smectite with $\mathrm{Na}^{+}$ions (VFA), the primary reflection of the clay gave stronger intensity and is shifted from $7.22^{\circ} 2 \theta$ to a lower diffraction angle $7.01^{\circ} 2 \theta$, which corresponds to an increase in the clay basal spacing to $12.6 \AA$.

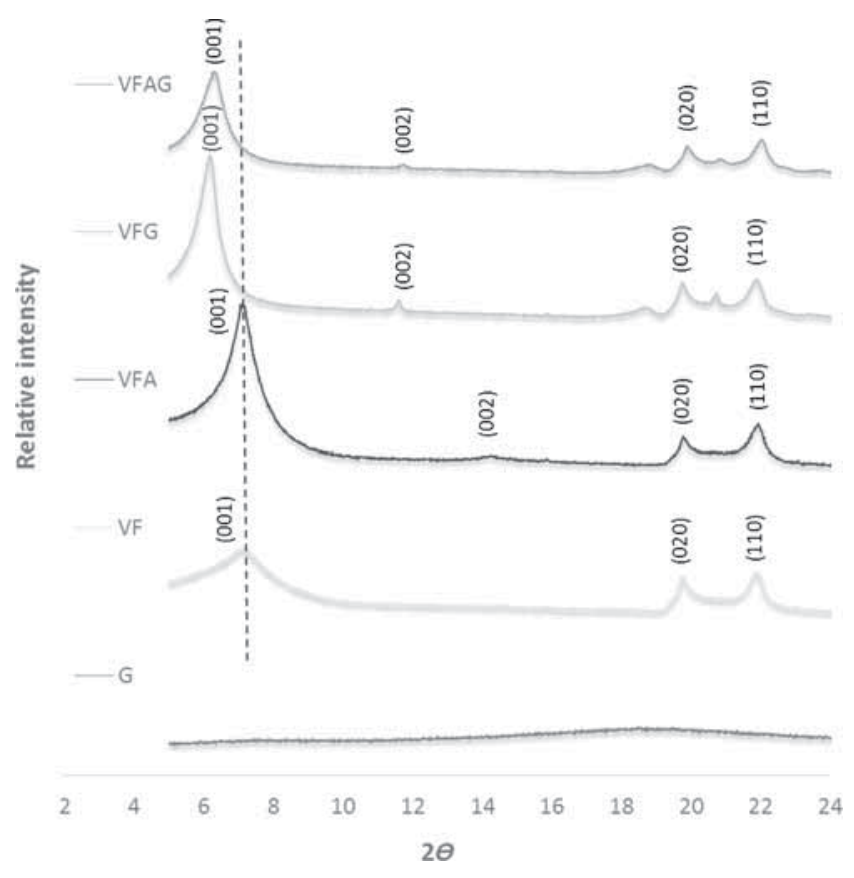

Figure 1. XRD patterns of the VF, VFA, VFG and VFAG hybrids.

Table 1. Interbasal distance of VF, VFA, VFG and VFAG hybrids.

\begin{tabular}{lcc}
\hline Powder & $d_{001}(\AA)$ & $2 \theta\left(^{\circ}\right)$ \\
\hline VF & 12.3 & 7.22 \\
VFA & 12.6 & 7.01 \\
VFG & 14.5 & 6.10 \\
VFAG & 13.9 & 6.32 \\
\hline
\end{tabular}

The basal spacing of the MAS hybrids intercalated with gentamicin reached plateau at $14.5 \AA$ which corresponds to the shifted principle reflection at $6.10^{\circ} 2 \theta$. The basal spacing of the MAS hybrids modified with $\mathrm{Na}^{+}$ions prior intercalation was slightly lower and reached $13.9 \AA$ at corresponding $6.32^{\circ} 2 \theta$ angle. Reflection peaks of the clay have increased in intensity for hybrid materials (VFG and VFAG $v s$. VF), when gentamicin-MAS complexes were created, and additional peaks also appeared in XRD patterns at $2 \theta=11.64^{\circ}, 18.91^{\circ}$ and $20.90^{\circ}$ as a consequence of structural changes appearing in the presence of gentamicin. The mentioned additional peaks were previously reported by others authors studying the intercalation of nicotine into magnesium aluminium silicate powders. ${ }^{9}$ Position of the principal peak of non-activated MAS powder (VF) shifts significantly after the introduction of gentamicin into the silicate-based system, and according to Bragg's law the peak shifting from higher diffraction angle to lower diffraction angle is due to increase in the $d$-spacing, which indicates that gentamicin has been effectively intercalated into the interlayer of MAS and is positioned flat on the surface of MAS as monolayer. ${ }^{18}$

The thickness of a single montmorillonite layer, which can be found using known crystallographic data ${ }^{19,20}$ or molecular simulations, is slightly lower than $7.0 \AA^{21,22}$ Since, $d_{(001)}$ is the sum of layer thickness and gallery height $\left(h_{\mathrm{g}}\right)$, the value of $h_{\mathrm{g}}$ can be easily calculated. Thus, the estimated gallery heights in VFA and VFAG silicates after intercalation, equaled approximately 7.6 and $6.0 \AA$, respectively. According to the size of the $\mathrm{G}$ molecule predicted by molecular modelling (length $15.3 \AA$ and height $5.2 \AA$ ), ${ }^{23}$ the values of interlayer spacing confirm, that gentamicin is located as a monolayer between two $2: 1$ montmorillonite layers together with a monolayer of water and most probably some sodium ions.

Since, the primary reflection was of stronger intensity for the VFG hybrid, compared to VFAG sample, and shifted towards lower values of the angle $2 \theta$, this corresponds to a greater separation of layers of the VFG powder and may indicate that a larger amount of gentamicin was intercalated into the layers of the unmodified powder (VF) than the modified powder (VFA) (VFG and VFAG after intercalation, respectively). However, the increased basal spacing after the interaction of gentamicin into the interlayer space of both smectite powders provides the evidence of the intercalation of the drug into the smectite clay, as it has been already mentioned.

\subsection{FTIR analysis}

The full FTIR spectra (in the range of $4000-400 \mathrm{~cm}^{-1}$ ) of the studied VF, VFA, VFG, VFAG and gentamicin powders are shown in figure 2. The FTIR spectrum of gentamicin sulphate shows absorption bands between 3500 and 3100 $\mathrm{cm}^{-1}$, which are characteristic for the occurrence of stretching vibration bands for $\mathrm{N}-\mathrm{H}$ amino groups. In the range between 3000 and $2800 \mathrm{~cm}^{-1}$, a large number of bands come from alkyl $(\mathrm{C}-\mathrm{H})$ stretching vibrations of gentamicin 


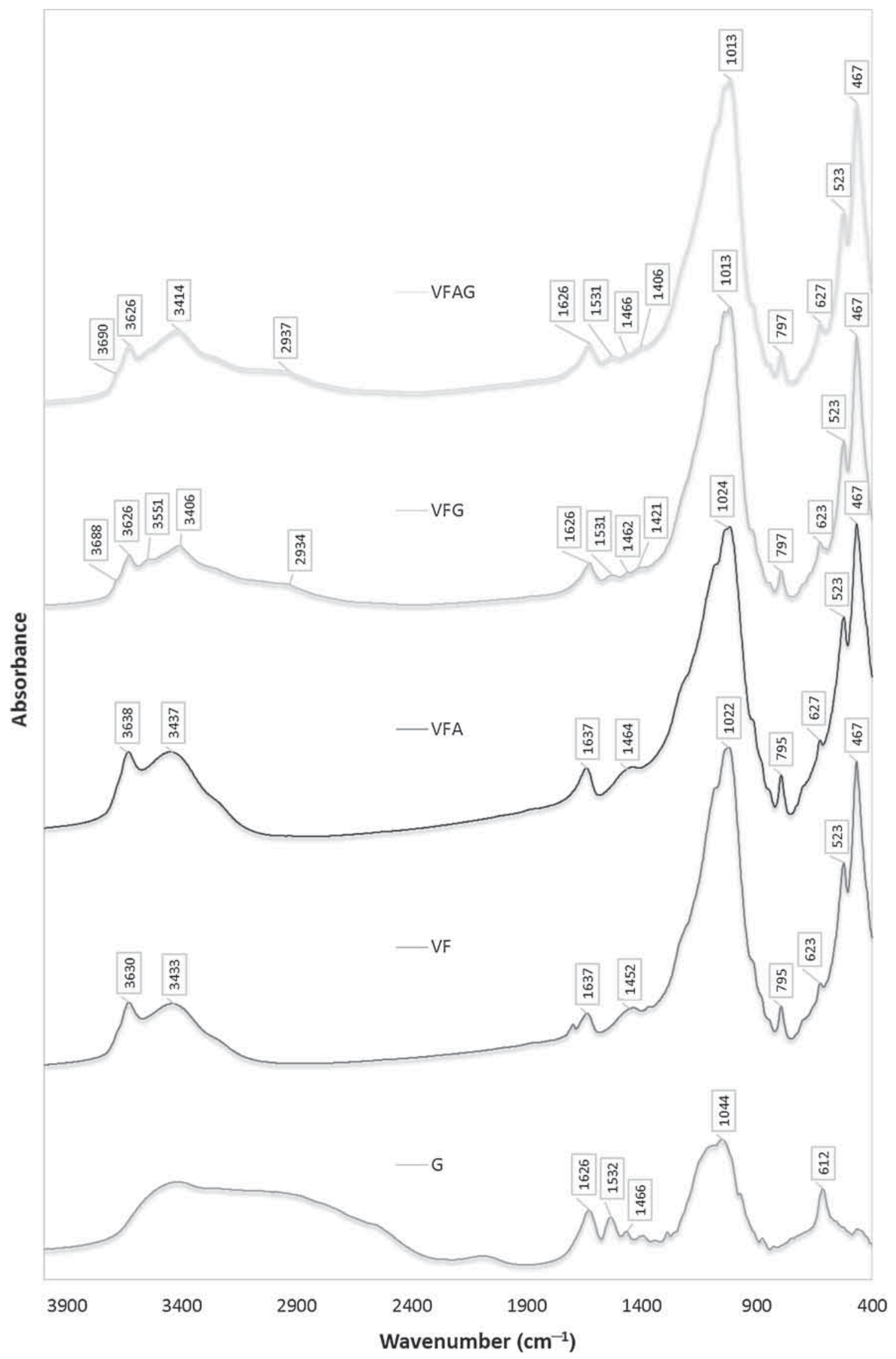

Figure 2. FTIR spectra of VF, VFG, VFA, VFAG and G powders. 
sulphate. Numerous molecules which are components of gentamicin sulphate contribute to the FTIR spectrum bands in the range $3600-2500 \mathrm{~cm}^{-1}$, thus the presence of a broad diffuse band can be explained. ${ }^{24}$ The subsequent bands at $1650-1400 \mathrm{~cm}^{-1}$ correspond to bending vibrations of $\mathrm{N}-\mathrm{H}$, stretching vibrations of $\mathrm{C}-\mathrm{H}$, and stretching vibrations of $\mathrm{C}-\mathrm{N}$. The strong absorption band between 1300 and $900 \mathrm{~cm}^{-1}$ corresponds to stretching vibrations of $\mathrm{C}-\mathrm{N}$ and also numerous stretching vibrations of group $\mathrm{C}-\mathrm{O} .^{24,25}$

The spectrum of the VF powder shows characteristic magnesium aluminium silicate bands, that is hydroxyl stretching of $\mathrm{Si}-\mathrm{OH}$ at $3630 \mathrm{~cm}^{-1}$, hydroxyl stretching of hydrogen bonded water at $3433 \mathrm{~cm}^{-1}$, hydroxyl bending at $1637 \mathrm{~cm}^{-1}$ and the stretching of $\mathrm{Si}-\mathrm{O}-\mathrm{Si}$ at $1022 \mathrm{~cm}^{-1}$. The absorption bands at 925 and $467 \mathrm{~cm}^{-1}$ are also visible in the spectrum and table 2 summarizes the band assignments for the MAS powder. ${ }^{8,10,26-32}$

In the FTIR spectra of the G-MAS hybrids (VFG and VFAG), compared to a pristine MAS and the MAS modified with $\mathrm{Na}^{+}$ions, a shift of the hydroxyl stretching band of water at $3433 \mathrm{~cm}^{-1}$ to a lower wavenumber (3406-3414 $\left.\mathrm{cm}^{-1}\right)$ is observed. This suggests the formation of intermolecular hydrogen bonding between water and gentamicin. ${ }^{33}$ The hydroxyl stretching band of $\mathrm{Si}-\mathrm{OH}$ at $3630 \mathrm{~cm}^{-1}$ has weaker intensity and is shifted to a lower wavenumber $\left(3626 \mathrm{~cm}^{-1}\right)$. This is accompanied with the hydrogen bonding formation of $\mathrm{Si}-\mathrm{OH}$ with an amine group of gentamicin and water bound with gentamicin acting as a water bridging mechanism. ${ }^{4}$ Additionally, the occurrence of the new peak at $3688-3690 \mathrm{~cm}^{-1}$, after the G-MAS hybrid formation, indicates free $\mathrm{OH}$ groups of the inner surface of the silicate layer of MAS. ${ }^{34}$ The stronger vibration of free hydroxyl groups on the inner surface of the silicate layers was enabled when the basal spacing of the MAS increased due to the intercalation of the gentamicin molecules. ${ }^{9}$ The band at $1652 \mathrm{~cm}^{-1}$ for the VF material, corresponding to bending vibrations of $\mathrm{OH}$, shifts its position to $1621 \mathrm{~cm}^{-1}$ for the VFG material. The decrease in intensity and shift

Table 2. FTIR bands of the VF powder. ${ }^{8,10,26-32}$

\begin{tabular}{lc}
\hline $\begin{array}{l}\text { Band position } \\
\left(\mathrm{cm}^{-1}\right)\end{array}$ & $\begin{array}{c}\text { Band assignment/ } \\
\text { description }\end{array}$ \\
\hline 3695 & Stretching vibrations O-H of Si-OH \\
3630 & Stretching vibrations O-H of Al-OH \\
3433 & Stretching vibrations O-H for adsorbed $\mathrm{H}_{2} \mathrm{O}$ \\
1700 & Bending vibrations H-O-H \\
1637 & Bending vibrations O-H for adsorbed $\mathrm{H}_{2} \mathrm{O}$ \\
1090 & Si-O stretching vibrations \\
1022 & Si-O stretching vibrations \\
925 & Bending vibrations Al-Al-OH \\
847 & Al-MgOH deformation \\
795 & Si-O stretching vibrations \\
624 & Al-OSi deformation \\
523 & Si-OSi deformation \\
467 & Coupled Al-O and Si-O out-of-plane vibrations \\
\hline
\end{tabular}

of the maximum of the peak originating from the vibrations of water molecules indicate a reduction in interlayer water content. These are again caused by the displacement of water molecules from the interlayer space by organic molecules derived from gentamicin.

In the VFG hybrid, the absorption band at $3551 \mathrm{~cm}^{-1}$ and characteristic sequence of bands at 1626, 1530 and 1466 $\mathrm{cm}^{-1}$ attributed to the presence of gentamicin are observed. The presence of new bands confirms that gentamicin interacts with the smectite layers and an additional band in the VFG spectrum at $3551 \mathrm{~cm}^{-1}$ is attributed to a stretching vibration band of $\mathrm{N}-\mathrm{H}$ amino groups of gentamicin. The $1532 \mathrm{~cm}^{-1}$ absorption band also comes from gentamicin and may be referred to stretching vibrations of $\mathrm{C}-\mathrm{H}$. The bending vibration $\mathrm{H}-\mathrm{O}-\mathrm{H}$ band disappears at the same time, which may be caused by the displacement of water from the interlayer space of the smectite. In the FTIR spectra of the GMAS hybrids (VFG and VFAG), a shift of the hydroxyl bending band of water at $1637 \mathrm{~cm}^{-1}$ to a lower wavenumber $1626 \mathrm{~cm}^{-1}$ is also observed, and its overlap with bending vibrations of $\mathrm{N}-\mathrm{H}$ coming from gentamicin, which may confirm the formation of intermolecular hydrogen bonding water and gentamicin.

In the FTIR spectrum for the VFG material, an increase in the intensity of the bands in the range $3300-2500 \mathrm{~cm}^{-1}$ is also evident. This is attributed to the presence of the stretching vibration band of $\mathrm{N}-\mathrm{H}$ amino groups and alkyl (C$\mathrm{H})$ stretching vibrations of gentamicin. ${ }^{35-38}$ Similar changes were observed in the FTIR spectrum for the VFAG hybrid, but the band at $3551 \mathrm{~cm}^{-1}$ is not present. Characteristic changes in the FTIR curves of both the VFG and VFAG materials in the range $3500-2500 \mathrm{~cm}^{-1}$ clearly shows that gentamicin molecules were introduced in the interlayer space of magnesium aluminium silicate. ${ }^{39}$

The activation process using $\mathrm{NaCl}$ solution introduces changes in the FTIR curve for the VFA powder. The FTIR spectrum of the VFA sample shows increases in the range between 4000 and $3200 \mathrm{~cm}^{-1}$ and $2100-1200 \mathrm{~cm}^{-1}$. The broad peak at $3442 \mathrm{~cm}^{-1}$ is intensified, which may be caused by changes in the interlayer spaces after ionic activation. ${ }^{35,36}$

It can be stated finally that both magnesium aluminium silicate-based materials, before and after ionic activation, can
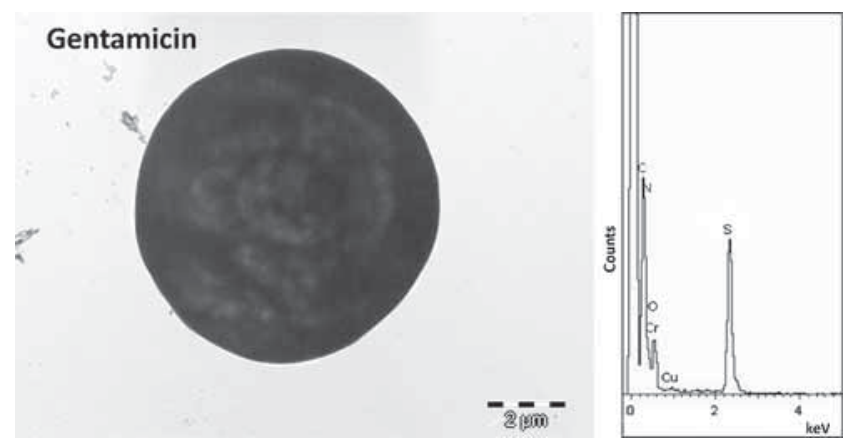

Figure 3. TEM micrograph and EDS analysis of gentamicin sulphate powder. 
be used as drug carriers. However, the summary of FTIR test results for the VFG and VFAG hybrids does not permit a clear statement as to which of these materials has a greater capacity to absorb the drug in the interlayer.

\subsection{TEM, SEM and EDS results}

A TEM micrograph of the gentamicin sulphate powder and its EDS analysis are presented in figure 3. Gentamicin particles were of a spherical shape with varying diameters. EDS analysis performed at several points of the gentamicin particles showed the presence of sulphur as an element identifying the powder. The presence of $\mathrm{Cu}$ and $\mathrm{Cr}$ in the EDS graph is associated with the substrate onto which the powder, in the form of an alcohol suspension, was applied prior to the microscopic observation.

The morphology and the average elemental analysis of the studied powder are shown in figure 4 . The starting powder (VF) was characterized by the 'flake-like' structure typical for smectite clay, forming agglomerates. EDS analysis showed the presence of elemental compositions typical for magnesium aluminium silicates; however, the analysis did not reveal the presence of sodium. After $\mathrm{NaCl}$ activation, the sodium peak eventually appears at each analysed point of the VFA powder. The typical structure of the smectite powders after intercalation with gentamicin (VFG and VFAG) was also preserved, however the platelets seem to be stacked more loosely. Average elemental analysis of both powders after introduction of gentamicin shows the presence of sulphur as an indicator of the presence of drug, but a higher peak of sulphur was observed for the VFG powder.

The SEM results also provide important information about the morphology of the MAS materials, before and after intercalation with gentamicin. SEM micrographs and elemental mapping of the VF and VFA powders are presented in figure 5. The initial magnesium aluminium silicate particles were in a form of the distinct sphere-like grains of the clay mineral, composed of many small flakes (figure 5a). Elemental mapping analysis made for the presence of the Na element, in the pressed VF powder, showed the presence of only a few points on the surface of the powder.

The change in the morphology of the magnesium aluminium silicate powder after $\mathrm{NaCl}$ activation can be seen in figure 5b. There is a clear 'house of cards' microstructure typical for the smectite, proving the separation of layers after activation. Elemental mapping analysis of the VFA powder showed sodium to be distributed homogeneously in the VFA matrix. This means that the activation process occurred successfully and the layers of MAS were consequently moved apart.

SEM micrographs, EDS and elemental mapping analysis of gentamicin sulphate are shown in figure 6. Gentamicin sulphate powder has a characteristic morphology consisting of spheres ranging in size from several to dozens of micrometres. EDS analysis, performed at two different points
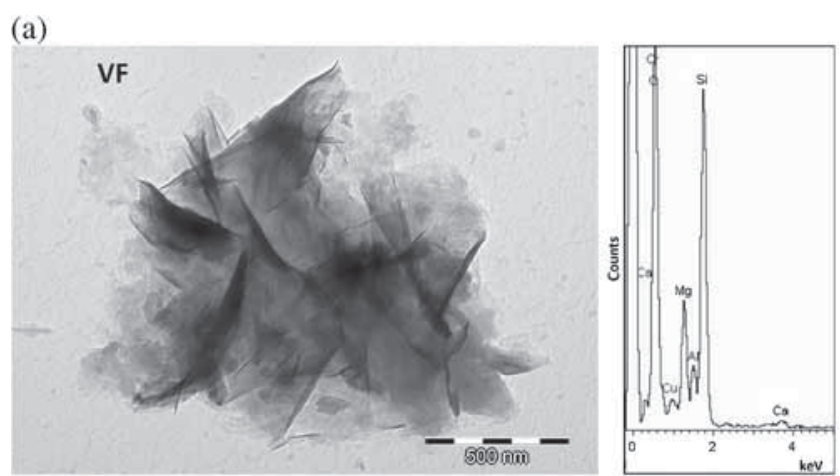

(b)
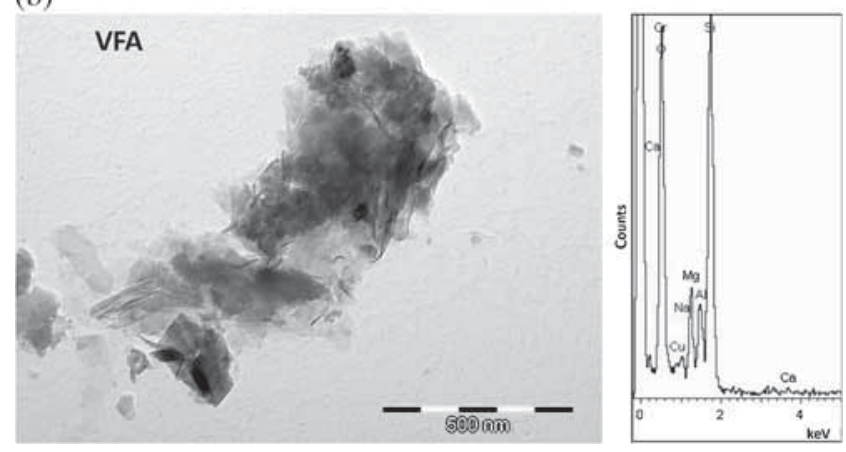

(c)
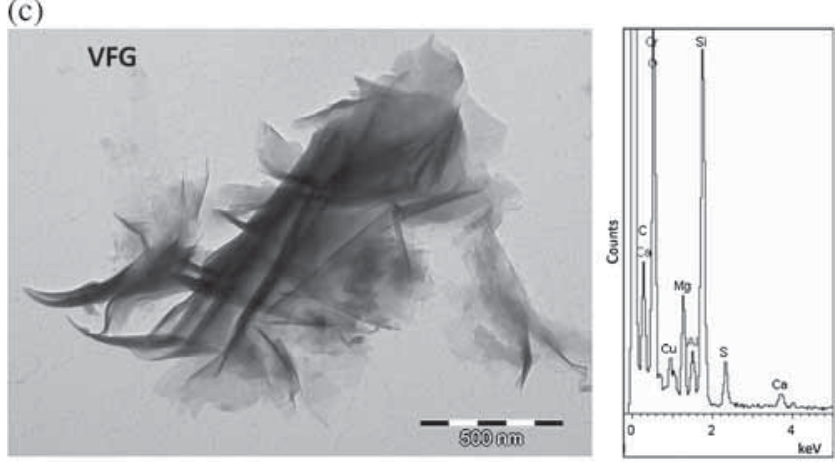

(d)
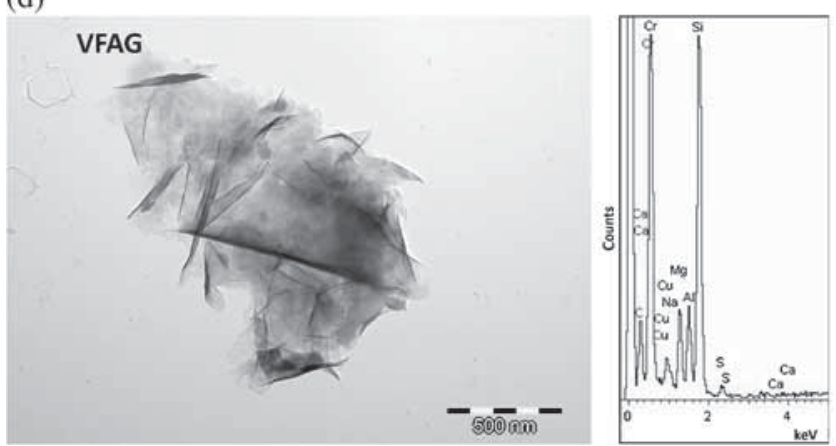

Figure 4. TEM micrographs and EDS analysis of the studied powders (VF, VFA, VFG and VFAG).

of the powder, shows that the major characteristic peak of gentamicin is a sulphur peak. The presence of homogeneously distributed sulphur in the gentamicin sulphate powder matrix was also confirmed by elemental mapping analysis. 
(a)
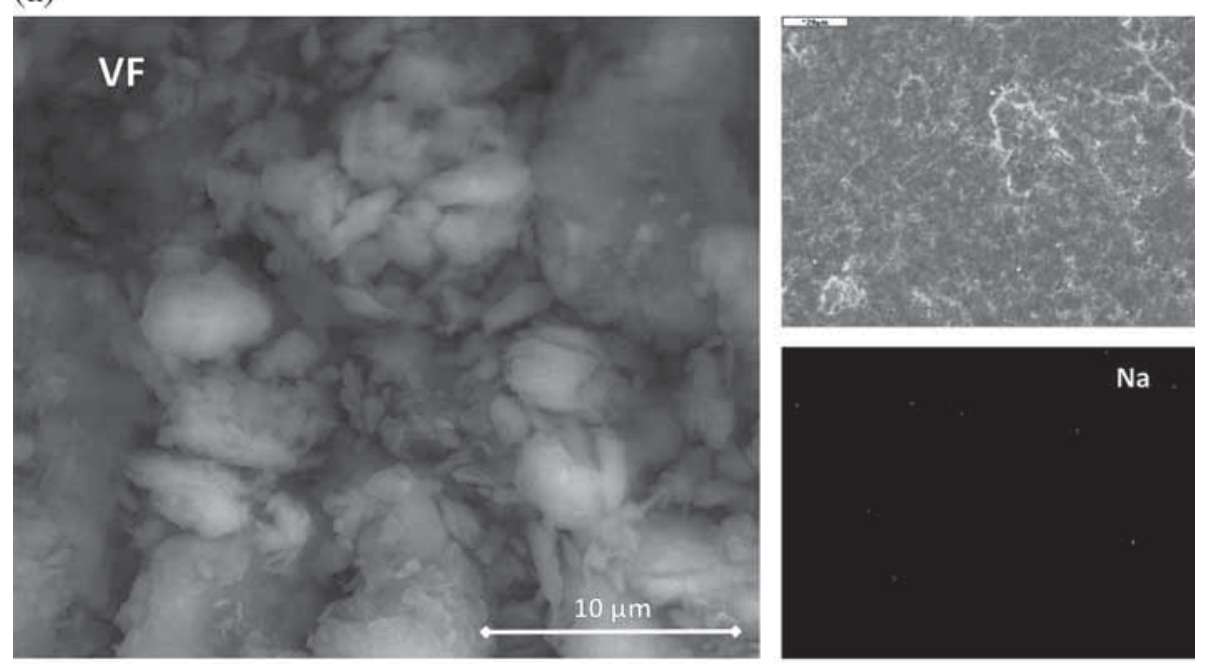

(b)
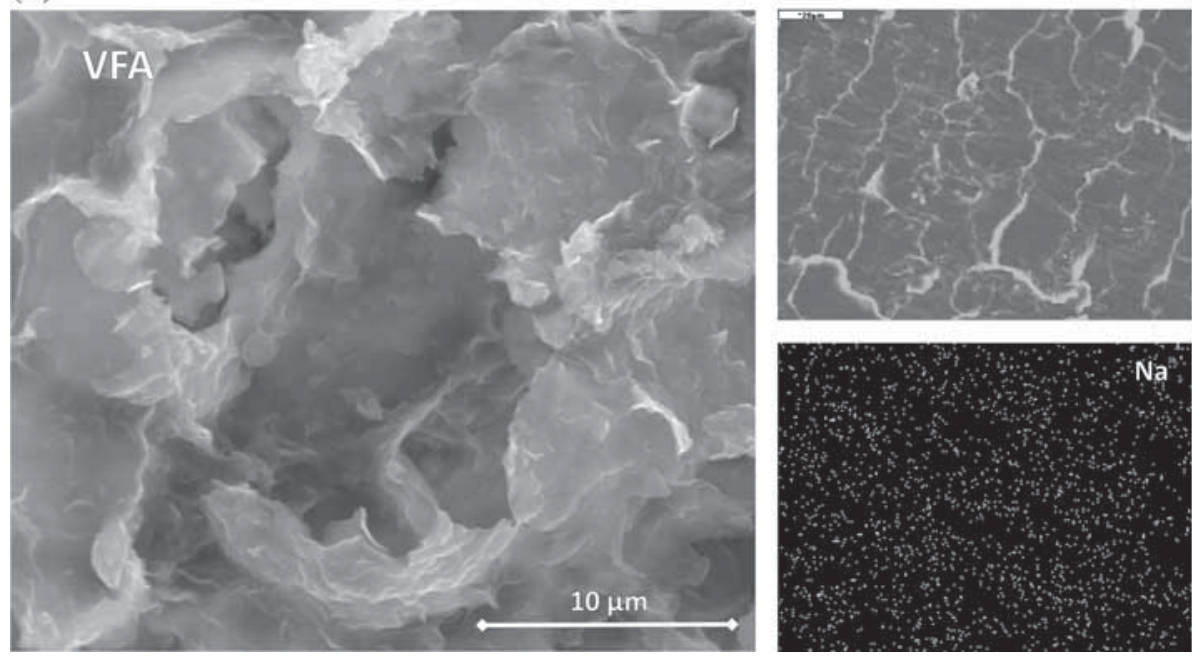

Figure 5. SEM micrographs and elemental mapping analysis of the (a) VF and (b) VFA powders.
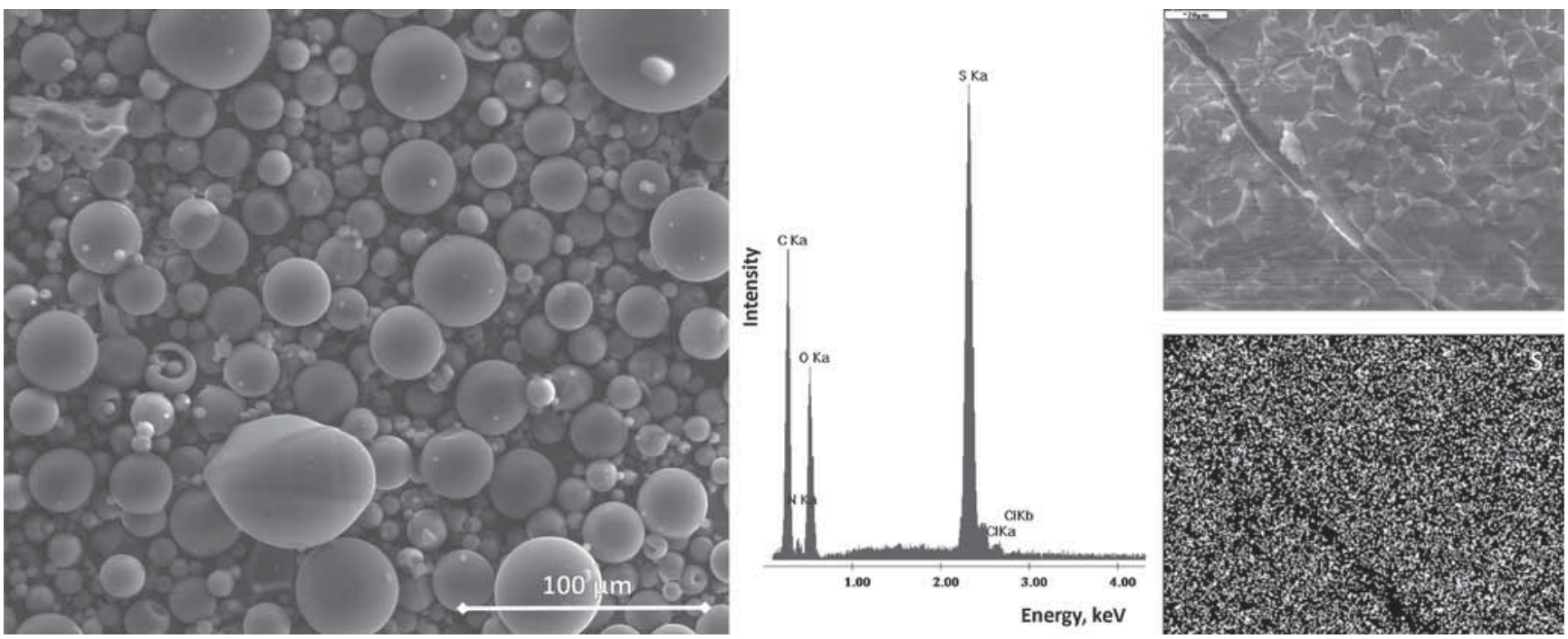

Figure 6. SEM micrographs, EDS and elemental analysis of gentamicin sulphate. 

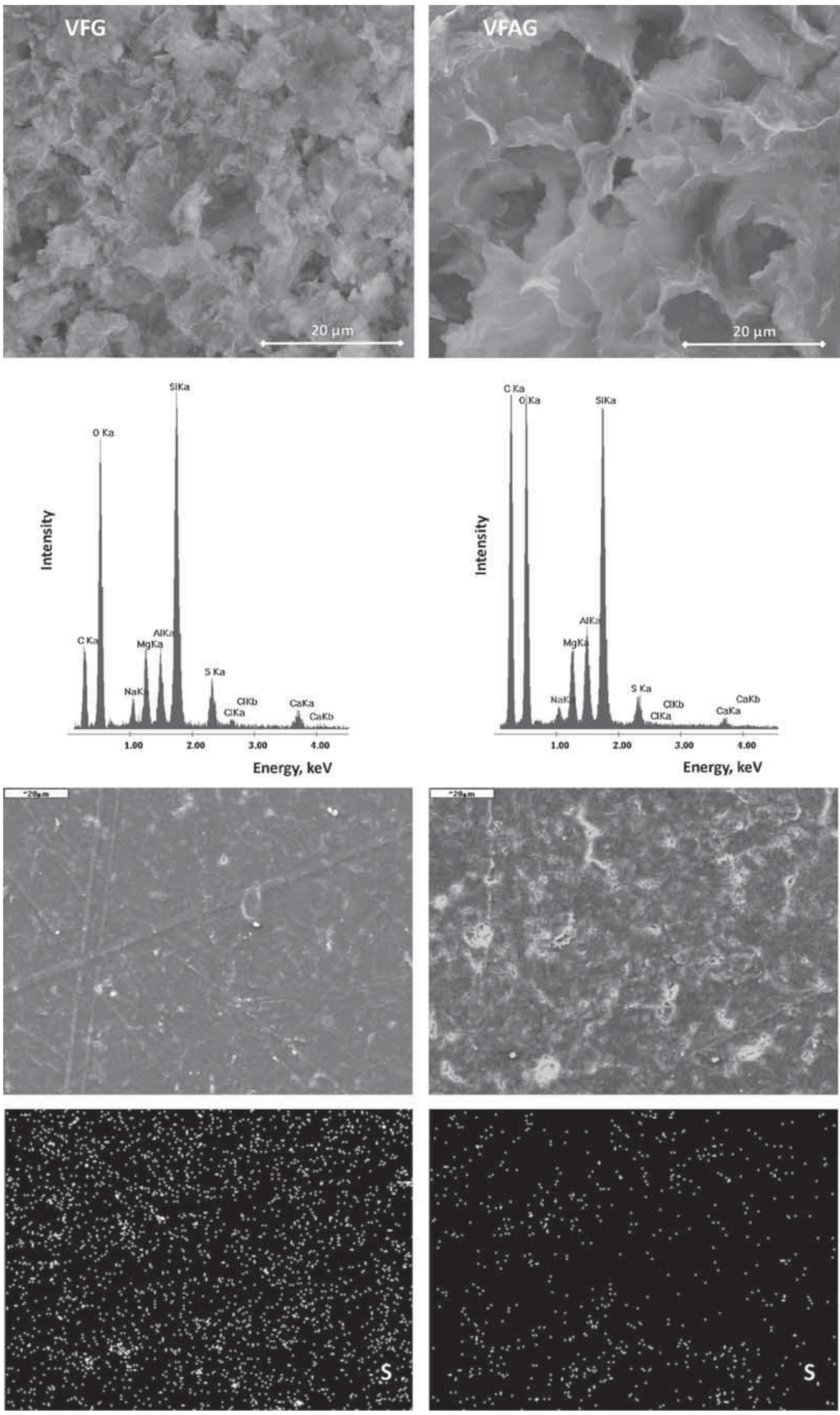

Figure 7. SEM micrographs, EDS and elemental mapping analysis of the VFG and VFAG powders. 
SEM micrographs, EDS and elemental mapping analysis of magnesium aluminium silicate after intercalation with gentamicin (VFG and VFAG) are shown in figure 7. Both powders after intercalation of gentamicin reveal the "house of cards' microstructure, however the appearance of the VF clay particle after intercalation with gentamicin changed significantly. The sphere-like particles of VF were replaced by high- and low-aspect ratio flakes of VFG, while the grain boundaries steadily disappeared. EDS analysis, carried out at several points for both powders VFG and VFAG, confirmed the presence of sulphur, which is a characteristic element of gentamicin. The EDS results indicated that sulphur is homogeneously distributed in the matrix powder of the (nonactivated) VFG sample. An apparent difference is also visible in the amount of sulphur in the samples of both powders after intercalation: the amount of sulphur in the VFG powder is higher and more evenly distributed compared with the amount and distribution of this element in the VFAG sample. It can therefore be concluded, on the basis of elemental mapping analysis of SEM and previously described XRD studies, that intercalation of clays with gentamicin is preferred in the case of unmodified smectite. Thus, the use of activated smectite as a drug carrier may be less favourable.

\section{Conclusions}

In this study, gentamicin, belonging to the aminoglycoside family of antibiotics, was successfully intercalated into both unmodified and modified (with $\mathrm{NaCl}$ ) magnesium aluminium silicates. The introduction of gentamicin between smectite clay layers was confirmed by XRD, FTIR, TEM and SEM analysis.

The presented set of research methods enabled confirmation of the intercalation of gentamicin into the interlayer of magnesium aluminium silicate. Characteristic changes in the FTIR curves of the MAS-based hybrid materials with gentamicin, both unmodified VFG and VFAG after prior modification with $\mathrm{Na}^{+}$cations, in the range of 1650-1400 and $3600-2500 \mathrm{~cm}^{-1}$, show that gentamicin molecules were introduced into the hybrid. The absorption band at 3551 $\mathrm{cm}^{-1}$ and characteristic sequence of bands at 1626, 1530 and $1466 \mathrm{~cm}^{-1}$, attributed to the presence of gentamicin, confirm that gentamicin interacts with the smectite layers. The shifted position of peaks at 3630,3433 and $1637 \mathrm{~cm}^{-1}$, as well as the presence of new bands at 3688 and $3551 \mathrm{~cm}^{-1}$ provide rather clear evidence, that gentamicin was bound in the structure of the magnesium aluminium silicate, thus intercalation of gentamicin has occurred.

The intercalation process of gentamicin was confirmed by XRD analysis and the XRD patterns of the VFG and VFAG powders showed that the primary reflection $d_{001}$ was of stronger intensity for the VFG hybrid and shifted from $7.22^{\circ} 2 \theta$ to significantly lower value of $6.10^{\circ} 2 \theta$, compared to $6.32^{\circ} 2 \theta$ measured for VFAG hybrid. This corresponds to a greater separation of layers of the VFG powder, which increased from 12.3 to $14.5 \AA$ after introduction of gentamicin, in comparison to a change from 12.6 to $13.9 \AA$ reported for the VFAG powder. It may indicate that a larger amount of gentamicin was intercalated into the layers of the unmodified powder VF than the modified powder VFA. However, the increased basal spacing after the interaction of gentamicin with both MAS powders provides a clear evidence of the intercalation of the drug, in a form of monolayer, into the MAS host material.

The TEM and SEM with EDS were also used to confirm the intercalation of smectite clay with gentamicin. The change in the physical appearance of the clay particles was observed and the sphere-like particles were replaced by high- and low-aspect flakes after the intercalation. The SEM elemental mapping analysis was also capable to provide the most complete information on homogeneity of the intercalated gentamicin and its distribution in the host clay material.

The results of instrumental characterization of the smectite clay-gentamicin hybrids indicate that the studied MAS clays can be potentially used as a sustained release carriers of gentamicin antibiotic. It was also shown, on the basis of XRD and SEM elemental mapping analysis, that nonactivated smectite clay is most probably a more suitable as the drug carrier of gentamicin.

\section{Acknowledgements}

Work was carried out within the framework of funding for statutory activities of AGH University of Science and Technology in Cracow, Faculty of Materials Science and Ceramics (11.11.160.617).

\section{References}

1. Dash A K and Cudworth II G C 1998 J. Pharmacol. Toxicol. Methods $\mathbf{4 0} 1$

2. López-Galindo A, Viseras C and Cerezo P 2007 Appl. Clay Sci. 3651

3. Lee Y H, Kuo T F, Chen B Y, Feng Y K, Wen Y R, Lin W C and Lin F H 2005 Biomed. Eng. Appl. Basis Commun.: Basis Commun. 1772

4. Aguzzi C, Cerezo P, Viseras C and Caramella C 2007 Appl. Clay Sci. 3622

5. Mostafavi A, Emami J, Varshosaz J, Davies N M and Rezazadeh M 2011 Int. J. Pharm. 409128

6. de Sousa Rodrigues L A, Figueiras A, Veiga F, Mendes de Freitas R, Nunes L CC, Cavalcanti da Silva Filho E and da Silva Leite C M 2013 Colloid Surf. B: Biointerfaces 103 642

7. Zheng J P, Luan H Y, Xi L F and Yao K D 2007 Appl. Clay Sci. 36297

8. Joshi G V, Patel H A, Kevadiya B D and Bajaj H C 2009 Appl. Clay Sci. 45248 
9. Pongjanyakul T, Khunawattanakul $\mathrm{W}$ and Puttipipatkhachorn S 2009 Appl. Clay Sci. 44242

10. Kevadiya B D, Joshi G V, Mody H M and Bajaj H C 2011 Appl. Clay Sci. 52364

11. Nunes C D, Vaz P D, Fernandes A C, Ferreira P, Roma C C and Calhorda M J 2007 Eur. J. Pharm. Biopharm. 66357

12. Park J K, Choy Y B, Oh J M, Kim J Y, Hwang S J and Choy J H 2008 Int. J. Pharm. 359198

13. Viseras C, Aguzzi C, Cerezo P and Lopez-Galindo A 2007 Appl. Clay Sci. $\mathbf{3 6} 50$

14. Kibbe H A 2000 Handbook of pharmaceutical excipients (Washington: American Pharmaceutical Association) 3rd ed

15. Rojtanatanya S and Pongjanyakul T 2008 Int. J. Pharm. 383 106

16. Darder M, Colilla M and Ruiz-Hitzky E 2003 Chem. Mater. 15 3774

17. Pongjanyakul T, Priprem A and Puttipipatkhachorn S $2005 \mathrm{~J}$. Control. Release 107343

18. Legaly G T and Dekany I 2005 Adv. Colloid Interface Sci. 114115189

19. Bailey S W (ed) 1988 Rev. Miner. 19189

20. Lepoittevin B, Devalckenaere M, Pantousier N, Alexandre M, Kubies D, Calberg C et al 2002 Polymer 434017

21. Kiersnowski A, Dabrowski P, Budde H, Kressler J and Piglowski J 2004 Eur. Polym. J. 402591

22. Katti K S, Sikdar D, Katti D R, Ghosh P and Verma D 2006 Polymer 47403

23. Doadrio A L, Sousa E M B, Doadrio J C, Perez Pariente J, Izquierdo-Barba I et al 2004 J. Control. Release 97125
24. Baia M, Astilean S and Iliescu T 2008 Raman and SERS investigations of pharmaceuticals (Berlin, Heidelberg: SpringerVerlag)

25. Leopold N, Cîntă-Pînzaru S, Baia M, Antonescu E, Cozar O, Kiefer W and Popp J 2005 Vibr. Spectrosc. 39169

26. Viscarra Rossel R A and Lark R M 2009 Eur. J. Soil Sci. 60453

27. Amarasinghe P M, Katti K S and Katti D R 2008 Appl. Spectrosc. 621303

28. Amarasinghe P M, Katti K S and Katti D R 2009 J. Colloid Interface Sci. $\mathbf{3 3 7} 97$

29. Joshi G V, Kevadiya B D, Patel H A, Bajaj H C and Jasra R V 2009 Int. J. Pharm. 37453

30. Patel H A, Somani R S, Bajaj H C and Jasra R V 2007 Curr. Sci. 921004

31. Ambre A, Katti K S and Katti D R 2011 Mater. Sci. Eng. C 31 1017

32. Nayak P S and Singh B K 2007 Bull. Mater. Sci. 30235

33. Raferty D W and Koenig J L 2002 J. Control. Release 8329

34. Hoch M and Bandara A 2005 Colloid Surf. A: Physicochem. Eng. Aspect. 253117

35. Madejová J 2003 Vibr. Spectrosc. 311

36. Xu W, Johnston C T, Parker P and Agnew S F 2000 Clays Clay Miner. 48120

37. Hongping H, Ray F L and Jianxi Z 2004 Spectrochim. Acta Part A: Mol. Biomol. Spectr. 602853

38. Madejowá J, Janek M, Komadel P, Herbert H-J and Moog H C 2002 Appl. Clay Sci. 20255

39. White J L and Hem S L 1983 Ind. Eng. Chem. Prod. Res. Dev. 22665 